Ann. Génét. Sél. anim., 1981, 13 (2), 75-92

\title{
Effets quantitatifs associés au gène albinos lié au sexe chez la Caille japonaise
}

\author{
P. MÉRAT, A. BORDAS, Françoise JONON \& A. PERRAMON \\ avec la collaboration technique de J.-L. Monvoisin \& J. Costa DA Silva \\ I.N.R.A., Laboratoire de Génétique factorielle \\ Centre de Recherches zootechniques \\ F 78350 Jouy-en-Josas
}

\begin{abstract}
Résumé
Deux années successives (1979 et 1980), des cailles femelles (Coturnix coturnix japonica), les unes de type sauvage, les autres, sœurs ou demi-sœurs des premières, portant le gène $s^{\text {al }}$ (albinos lié au sexe), ont été comparées pour la survie en fin d'incubation et après l'éclosion, l'activité en open-field, et divers paramètres quantitatifs mesurant la croissance pondérale, la production et les qualités des oufs, l'efficacité alimentaire en cages individuelles, enfin l'état du plumage des pondeuses après transfert en cages collectives.

Les albinos comparées aux cailles pigmentées présentent une mortalité nettement plus élevée après l'éclosion. Ce phénomène se limite aux premières semaines d'élevage et disparaît après 5 semaines d'âge. Leur croissance initiale est retardée, mais l'écart, en valeur absolue et encore plus en pourcentage du poids moyen des deux génotypes, diminue jusqu'au stade adulte (93 jours). Les cailles albinos à 5 semaines sont très significativement moins «actives 》 (temps de latence et nombre de tops). Le nombre et la masse totale d'œufs pondus en 28 jours ne diffère pas pour les génotypes «albinos» et «sauvage», non plus que l'âge au $1^{\text {er }}$ œuf. Par contre, la consommation alimentaire des pondeuses albinos est d'environ 6 p. 100 inférieure à celle des cailles sauvages. Exprimée en écart à la régression multiple sur le poids corporel, la variation de poids et la masse d'œufs pondus, cette consommation reste d'environ 4 p. 100 moindre pour le génotype [sal]. Le poids moyen des œufs est légèrement plus faible et le pourcentage de jaune supérieur pour ce génotype. Enfin, après 2 mois passés en cages de groupe, les cailles albinos ont un plumage en meilleur état que leurs parentes normalement pigmentées, ce qui, vraisemblablement, devrait augmenter la différence d'efficacité alimentaire entre les deux génotypes par rapport au résultat obtenu en cages individuelles.
\end{abstract}

\section{Introduction}

Chez la Caille japonaise (Coturnix coturnix japonica), un gène récessif lié au sexe, responsable d'un albinisme imparfait (symbole $s^{\text {al }}$ ) au locus $\mathrm{s}=$ albinos lié au sexe, a été décrit par Lauber (1964) et Homma et al. (1968). Chez la Poule, un gène analogue, également lié au sexe, est connu (Muellet \& Hutt, 1941 ; Werret et al., 1959).

Il ne semble pas que l'on ait recherché chez la Caille les répercussions possibles de ce mutant sur des caractères quantitatifs. Chez la Poule, à défaut de mesures des performances, plusieurs auteurs rapportent des impressions ou observations quali- 
tatives. Mueller \& Hutt (1941), puis Champion (1958) ne constatent pas de différence évidente entre oiseaux albinos ou non pour le taux d'éclosion, la survie des jeunes, la ponte. HuTt \& COLE (1973) concluent également à une vitalité normale des albinos. Selon Werret et al. (1959) les poussins albinos sont de petite taille à la naissance et paraissent avoir une croissance plus lente que les autres. D'autres observations, également non chiffrées, concernent le duvet plus court des albinos (Champion, 1958 ; Hutt \& COLE, 1973) et une sensibilité particulière à de fortes intensités lumineuses (Hurr \& CoLE, 1973) ou même une vision défectueuse (WERRET et al., 1959).

Notre population de cailles japonaises contenant le mutant albinos lié au sexe, il nous a paru intéressant de comparer des cailles albinos et de type sauvage, issues des mêmes reproducteurs, pour divers caractères quantitatifs liés à la production (croissance et ponte), à la survie et au comportement.

\section{Matériel et méthodes}

\section{Matériel animal et schéma expérimental}

Les cailles utilisées étaient issues du croisement de mâles hétérozygotes pour le gène albinos lié au sexe et de femelles hémizygotes de type sauvage, suivant le schéma :

$$
s^{+} s^{\mathrm{al}} \times \subsetneq s^{+}
$$

Les parents provenaient du troupeau expérimental du laboratoire, dont certaines familles présentaient une ségrégation au locus albinos.

Ce croisement était destiné à produire en proportion égale, aux fluctuations aléatoires près, des filles des deux génotypes hémizygotes $s^{+}$(type sauvage) et $s^{\text {al }}$ (albinos). Chaque mâle était apparié à deux femelles dans une cage pedigree. Les œufs, puis les jeunes à l'éclosion, étaient identifiés d'après le père seul. Chaque individu recevait à la naissance une bague de patte, puis à 14 jours une bague d'aile. Les filles seules étaient gardées, après identification du sexe par la pigmentation du plumage de la poitrine. Dans toute la mesure du possible, elles étaient choisies par paires de sœurs ou demisœurs, l'une de type sauvage, l'autre albinos.

Deux expériences étaient réalisées, l'une en 1979, l'autre en 1980. La première, avec 19 pères, comportait une éclosion unique, le 31 mars, à partir d'œufs collectés pendant deux semaines. La seconde, avec 27 pères, comprenait deux éclosions du même type, le 23 janvier et le 5 mars.

\section{Conditions d'élevage}

A la sortie de l'éclosoir, les cailleteaux étaient mis dans une batterie chaude de 5 étages, à une température ambiante de $38^{\circ} \mathrm{C}$ pendant les premiers jours, diminuant progressivement jusqu'à $30^{\circ} \mathrm{C}$ à l'âge de 15 jours, et maintenue entre 25 et $30^{\circ} \mathrm{C}$ de 15 jours à 5 semaines. 
A 5 semaines d'âge, les femelles gardées en vue de la phase de ponte, étaient réparties au hasard dans une batterie de cages individuelles de 4 étages, à une température ambiante fluctuant autour d'une valeur moyenne voisine de $20^{\circ} \mathrm{C}$. L'entrée en ponte avait lieu en moyenne entre 6 et 7 semaines d'âge. Les cailles étaient gardées dans ce local jusqu'à l'âge de 13 semaines.

La durée journalière d'éclairement (lumière artificielle) était de $24 \mathrm{~h}$ pendant les trois premiers jours puis, en raison de contraintes liées au local, de $9 \mathrm{~h}$ jusqu'à la mise en cages individuelles à 5 semaines. A partir de cet âge, une lumière continue était de nouveau fournie (naturelle pendant le jour, artificielle la nuit).

L'aliment consommé pendant les deux premières semaines contenait 28 p. 100 de protéines totales et environ $2600 \mathrm{Kcal}$ ou 10,9 M.J./ $\mathrm{kg}$ E.M. Il était donné sous forme de farine. Sa formule était identique de 2 à 5 semaines d’âge, mais il était distribué sous forme d'un mélange en proportion égale de granulés et de farine. A partir de 5 semaines, l'aliment était donné entièrement sous forme de granulés, avec 16 p. 100 de protéines totales, $2600 \mathrm{Kcal} / \mathrm{kg}$ E.M., et 3,2 p. 100 de calcium.

$\mathrm{Du}$ point de vue sanitaire, aucun traitement particulier n'était appliqué durant l'expérience de 1979 où la mortalité était faible et l'état de santé des animaux apparemment satisfaisant. En 1980, à la suite d'une mortalité élevée dans les premières semaines d'élevage (plus de 30 p. 100 jusqu'à 4 semaines) pour la première éclosion, une infection par Salmonella Typhimurium a été diagnostiquée (*). En conséquence, les animaux de ce lot ont reçu un antibiotique dans l'eau de boisson de 22 à 33 jours d'âge ; les œufs du second lot ont été désinfectés par trempage et les cailleteaux ont reçu un antibiotique jusqu'à 10 jours d'âge. A la suite de ces traitements, la mortalité après l'âge de 4 semaines a été très faible pour le premier lot, et son niveau à tous âges était normal dans le second.

\section{Mesures et observations}

Le phénotype «albinos» $\left[s^{\mathrm{al}}\right]$ était déterminé visuellement à l'éclosion et sur les embryons morts dans les derniers jours d'incubation en 1980.

Les mesures faites sur jeunes, puis sur femelles en ponte, sont indiquées dans le tableau 1.

Toutes les pesées étaient faites le matin. Les deux dernières (65 et 93 jours) correspondaient respectivement au début et à la fin de la période de contrôle simultané de la production d'œufs et de la consommation alimentaire (cf. plus loin). Les femelles étaient mises à jeun depuis la veille à 17 heures; il n'était pas fait de corrections pour la présence éventuelle d'un œuf dans l'oviducte au moment de la pesée.

Les mesures en open-field sont faites à l'âge de 5 semaines à l'aide de l'appareil décrit par FAURE et Folmer (1975). Il s'agit d'une cuve circulaire dans laquelle six faisceaux lumineux dirigés vers 6 cellules photoélectriques permettent, pour chaque

(*) Autopsies faites à l'Ecole Nationale Vétérinaire d'Alfort ( $\mathbf{P}^{r}$ LE COANET). 
oiseau pris individuellement, d'enregistrer le temps mis pour couper un premier faisceau (latence), puis le nombre total de faisceaux interceptés pendant 100 secondes (nombre de « tops»).

\section{TABLEAU 1}

\section{Désignation des mesures et unités employées}

Measurements and units

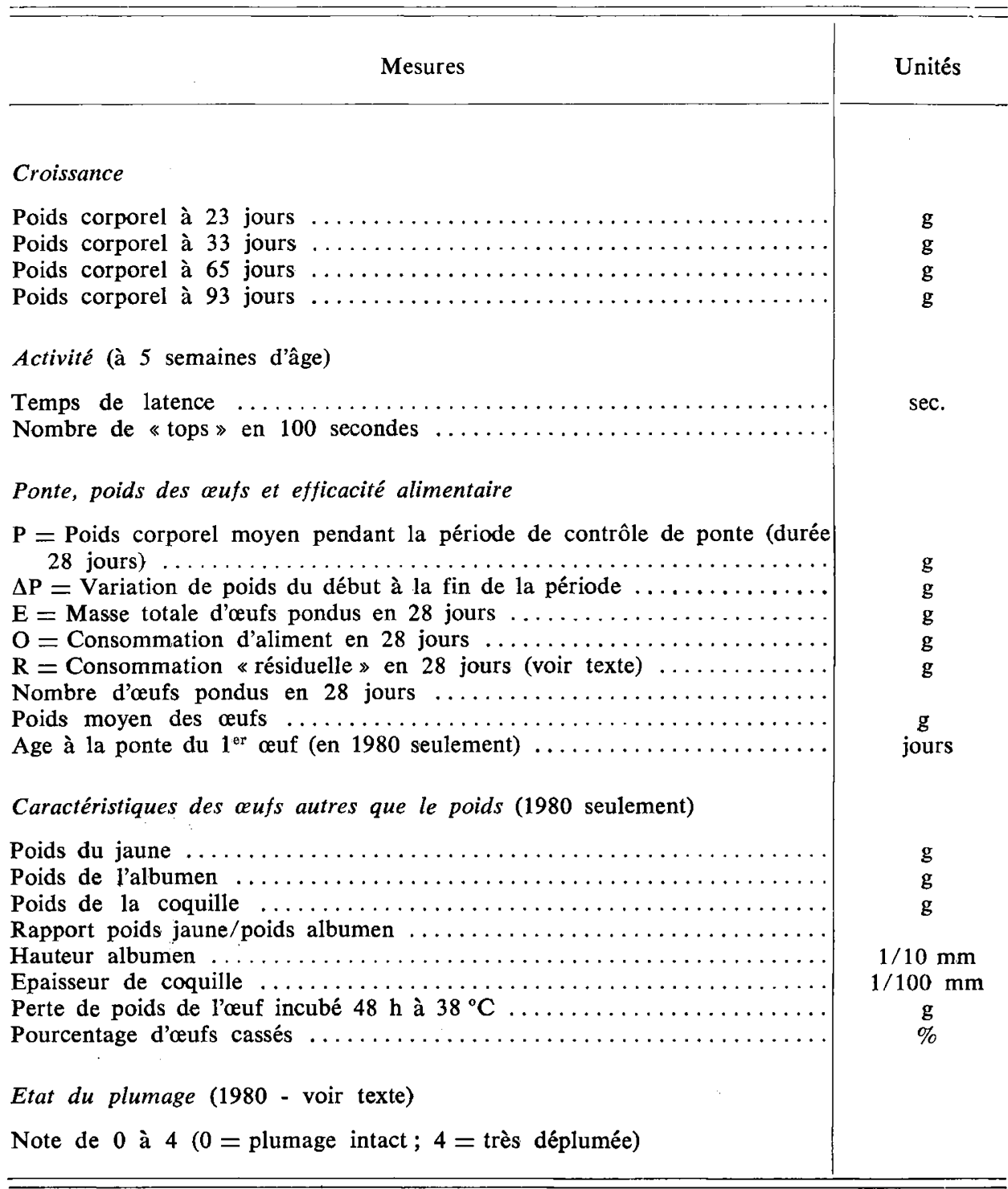


En ce qui concerne la phase de ponte, l'âge à la ponte du $1^{\text {er }}$ œuf n'était enregistré qu'en 1980. Puis, les deux années, une période de 28 jours, comprise entre les âges de 65 et de 93 jours, était choisie pour l'enregistrement des mesures relatives à la production d'œufs (toutes les femelles étant entrées en ponte) et à la consommation alimentaire. A partir des quatre variables $P$ (poids corporel moyen), $\Delta P$ (variation de poids), $\mathrm{E}$ (masse d'œufs pondue) et $\mathrm{O}$ (consommation d'aliment) relatives à cette période, une équation de régression multiple de la dernière variable sur les trois premières est établie. Cette équation est du type décrit par BYERLy (1941) puis d'autres auteurs chez la Poule :

$$
\mathbf{T}=\mathrm{aP} \boldsymbol{a}+\mathrm{b} \Delta \mathbf{P}+\mathrm{cE}+\dot{\mathbf{D}}
$$

où $\mathbf{T}$ est la consommation alimentaire prédite à partir des trois autres variables et $\mathbf{D}$ un terme constant. La valeur 0,5 était prise pour l'exposant $\alpha$, cette valeur ayant été trouvée voisine de l'optimum pour lequel la fraction de la consommation d'aliment expliquée par l'équation de régression est la plus grande. Nous avons établi une équation par génotype pour chaque année (et par lot d'éclosion en 1980). Après vérification de l'absence de différence significative entre génotypes intra-année ou éclosion quant aux coefficients de régression, les équations suivantes communes aux deux génotypes ont été retenues (1) :

$T=63,96 \mathrm{P}^{0,5}+2,63 \Delta \mathrm{P}+0,99 \mathrm{E}-267$
$\mathrm{~T}=20,22 \mathrm{P}^{0,5}+2,08 \Delta \mathrm{P}+1,41 \mathrm{E}+208$
$\mathrm{~T}=67,21 \mathrm{P}^{0,5}+2,18 \Delta \mathrm{P}+1,13 \mathrm{E}-355$ pour $1979\left(R^{2}=0,56\right)$

pour 1980, respectivement lots 1 et 2

$\left(\mathrm{R}^{2}=0,60\right.$ et 0,65$)$

C'est à partir de ces équations que, pour chaque individu, est calculée la différence $(R)$ entre sa consommation observée d'aliment $(\mathrm{O})$ et sa consommation théorique $(\mathrm{T})$.

Le poids moyen des œufs (sur les 28 jours de contrôle) est la seule caractéristique de ces derniers qui soit mesurée les deux années. Les autres variables, mesurées seulement en 1980, portent respectivement sur un œuf prélevé pendant la troisième semaine de contrôle (poids des composantes, jaune, albumen et coquille et rapport poids de jaune/poids d'albumen) et sur deux œufs pris pendant la même semaine (hauteur d'albumen étalé sur une surface plane, épaisseur de la coquille incluant la membrane coquillière). Sur deux autres œufs pris lors de la dernière semaine de contrôle est déterminée la perte de poids en 48 heures à $38^{\circ} \mathrm{C}$. Le pourcentage d'œufs cassés est établi sur les 28 jours de contrôle.

Enfin, en 1980, après la fin du contrôle des performances en cages individuelles, les femelles ont été placées pendant environ deux mois (depuis fin juin jusqu'au 5 septembre) dans trois batteries collectives. L'une recevait 34 cailles de type sauvage choisies au hasard, une seconde 34 femelles albinos; dans la troisième étaient placées 17 femelles de type sauvage et 17 albinos. La seule observation effectuée était, le 5 septembre, l'évaluation à vue, sur les survivantes, de l'état du plumage (plus ou moins détérioré avec zones dénudées) par une note de 0 à 4 .

(1) Les coefficients de ces trois équations, en fait, ne présentent pas de différences significatives; la faible valeur du coefficient de $\mathbf{P}^{0,5}$ pour le lot 1 de 1980 provient apparemment de l'échantillonnage, l'effectif de ce lot étant restreint. 


\section{Analyse statistique des données}

L'objet du présent article était la comparaison des femelles des deux génotypes «sauvage » $\left(s^{+}\right)$et «albinos» $\left(s^{\mathrm{al}}\right)$ pour les diverses variables enregistrées. Nous avons indiqué plus haut que, dans la mesure du possible, les femelles gardées après la détermination du sexe étaient choisies par paires de demi-sœurs ou sœurs, l'une de type sauvage, l'autre albinos, à l'intérieur de chaque année, et de chaque éclosion pour 1980. Pour chaque variable, seuls ceux de ces couples dont les deux membres étaient mesurés ont été pris en considération, et toutes les comparaisons entre génotypes sont faites par un test $t$ suivant la méthode des couples. Les valeurs moyennes présentées sont basées sur ces couples. Le détail en est donné pour chaque année, mais non pour chacune des deux éclosions en 1980.

Pour la mortalité embryonnaire en 1980, la comparaison entre génotypes est faite par un test de $\chi^{2}$. Il en est de même de la note d'emplumement en batteries collectives, après regroupement en deux classes d'importance à peu près comparable.

\section{Résultats}

La mortalité pa: génotype en 1980, sur les embryons identifiables après le $14^{\mathrm{e}}$ jour d'incubation, figure au tableau 2.

\section{Tableau 2}

Mortalité en fin d'incubation comparée pour les embryons sauvages et albinos Mortality at the end of the incubation period compared for wild-type and albino embryos

\begin{tabular}{c|c|c|c|c}
\hline \hline & $\begin{array}{c}\text { Nombre d'œufs } \\
\text { non éclos }\end{array}$ & $\begin{array}{c}\text { Nombre d'embryons } \\
\text { albinos morts }\end{array}$ & $\begin{array}{c}\text { Nombre d'embryons } \\
\text { sauvages morts }\end{array}$ & $\chi^{2}$ \\
\cline { 2 - 5 } Lot $1 \ldots$ & 18 & 29 & $4,42^{* *}$ \\
Lot $2 \ldots$ & 47 & 13 & 30 & 0,63 \\
\hline Total ... & 43 & 31 & 59 & 3,79 \\
\hline
\end{tabular}

: Significatif au seuil 1 p. 100.

La mortalité des jeunes n'a pas été relevée par génotype en 1979. En 1980, il n'en a pas été tenu compte pour la première éclosion, victime pendant les 3 à 4 premières semaines d'une mortalité anormale. Pour la seconde éclosion, la mortalité de 0 à 22 jours est respectivement pour les cailleteaux albinos et sauvages de 34,0 p. 100 et 1,2 p. 100 avec un maximum le $4^{e}$ jour; les valeurs correspondantes pour la période de 22 à 34 jours d'âge sont 8,3 p. 100 et 0,4 p. 100. Après cet âge, et tant en 1979 qu'en 1980, la mortalité est faible dans les deux génotypes. 
Tableau 3

Poids corporel à divers âges: comparaison sur des couples de femelles albinos et sauvages Body weight at 4 ages : comparison on pairs of albino and wild-type females

\begin{tabular}{|c|c|c|c|c|}
\hline & \multicolumn{4}{|c|}{ Ages (jours) } \\
\hline & 23 & 33 & 65 & 93 \\
\hline \multicolumn{5}{|l|}{1979} \\
\hline Nombre de couples . . & 44 & 41 & 32 & 32 \\
\hline $\begin{array}{l}\text { Poids moyen }(\mathrm{g}): \\
\mathrm{s}^{+} \quad \ldots \ldots \ldots \ldots \ldots \ldots \ldots \ldots \ldots \ldots \ldots \ldots \ldots\end{array}$ & $\begin{array}{r}109,43 \\
95,91\end{array}$ & $\begin{array}{l}153,34 \\
144,49\end{array}$ & $\begin{array}{l}165,13 \\
166,75\end{array}$ & $\begin{array}{l}178,47 \\
179,91\end{array}$ \\
\hline t et signification ... & $3,44 * *$ & $2,92 * *$ & 0,40 & 0,37 \\
\hline \multicolumn{5}{|l|}{1980} \\
\hline Nombre de couples... & 60 & 58 & 56 & 54 \\
\hline $\begin{array}{c}\text { Poids moyen }(\mathrm{g}): \\
\mathrm{s}^{+} \quad \ldots \ldots \ldots \ldots \ldots \ldots \ldots \ldots \ldots \ldots \ldots \ldots \\
\mathrm{s}^{\mathbf{a l}} \quad \ldots \ldots \ldots \ldots \ldots \ldots\end{array}$ & $\begin{array}{r}105,54 \\
91,60\end{array}$ & $\begin{array}{l}158,83 \\
142,02\end{array}$ & $\begin{array}{l}175,91 \\
163,87\end{array}$ & $\begin{array}{l}178,44 \\
169,75\end{array}$ \\
\hline$t$ et signification $\ldots . .$. & $5,16^{* * *}$ & $4,82 * * *$ & $3,41^{* *}$ & $2,45^{*}$ \\
\hline \multicolumn{5}{|l|}{ Total } \\
\hline Nombre de couples .... & 104 & 99 & 88 & 86 \\
\hline Différence moyenne $(\mathrm{g}) \ldots \ldots$ & 13,74 & 13,51 & 7,07 & 4,79 \\
\hline $\mathrm{t}$ et signification $\ldots \ldots \ldots \ldots$ & $6,08 * * *$ & $5,58 * * *$ & $2,55^{*}$ & 1,90 \\
\hline
\end{tabular}

$*_{-} * *_{-} * * *:$ Significatif respectivement au seuil 5,1 et 0,1 p. 100

Le tableau 3 indique les poids corporels successifs par génotype et année ainsi qu'au total et les tests de signification correspondants. Une présentation semblable est faite respectivement dans le tableau 4 pour les deux paramètres d' " activité », dans le tableau 5 pour les mesures relatives à la ponte, à la consommation et à l'efficacité alimentaire, dans le tableau 6 (année 1980 seulement) pour l'âge au premier œuf et les caractéristiques des œufs autres que le poids. 


\section{TABLEAU 4}

Mesures d'activité en open-field:

comparaison sur des couples de cailles femelles albinos et sauvages

Activity in open-field : comparison on pairs of albino and wild-type females

\begin{tabular}{|c|c|c|}
\hline & Latence (secondes) & Nombre de tops \\
\hline \multicolumn{3}{|l|}{1979} \\
\hline Nombre de couples $\ldots \ldots \ldots \ldots \ldots$ & 41 & 41 \\
\hline \multicolumn{3}{|l|}{ Valeur moyenne : } \\
\hline$s+\ldots$ & 26,51 & 8,85 \\
\hline $\mathrm{s}^{\mathrm{al}}$. & 56,44 & 5,29 \\
\hline$t$ et signification $\ldots .$. & $-3,52 * * *$ & $+1,41$ \\
\hline \multicolumn{3}{|l|}{1980} \\
\hline Nombre de couples & 58 & 58 \\
\hline \multicolumn{3}{|l|}{ Valeur moyenne : } \\
\hline$s+$ & 25,33 & 22,74 \\
\hline $\mathrm{s}^{\mathrm{al}}$ & 52,53 & 10,13 \\
\hline$t$ et signification & $-3,20 * *$ & $+2,80^{* *}$ \\
\hline \multicolumn{3}{|l|}{ Total } \\
\hline Nombre de couples & 99 & 99 \\
\hline Différence moyenne $\ldots \ldots \ldots \ldots$ & $-28,36$ & 8,87 \\
\hline$t$ et signification & $-5,03 * * *$ & $+3,32 * * *$ \\
\hline
\end{tabular}

$* *_{-} * * * ;:$ Significatif respectivement au seuil 5,1 et 0,1 p. 100 .

Le tableau 7 donne les pourcentages de cailles légèrement ou non déplumées (note 0 à 2) ou fortement déplumées (note 3 ou 4) suivant le génotype et le mode de groupement. 


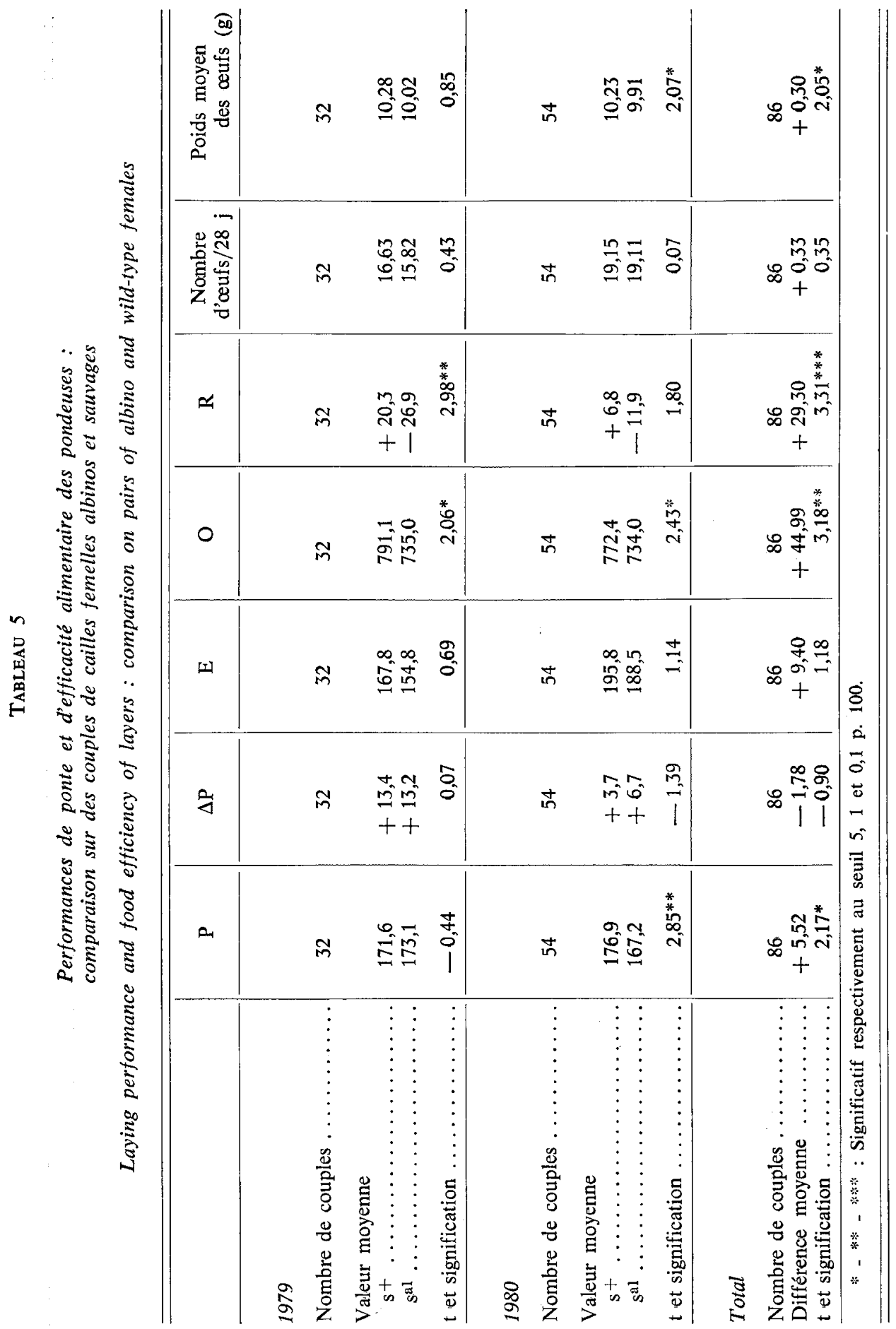


P. MÉRAT et al.

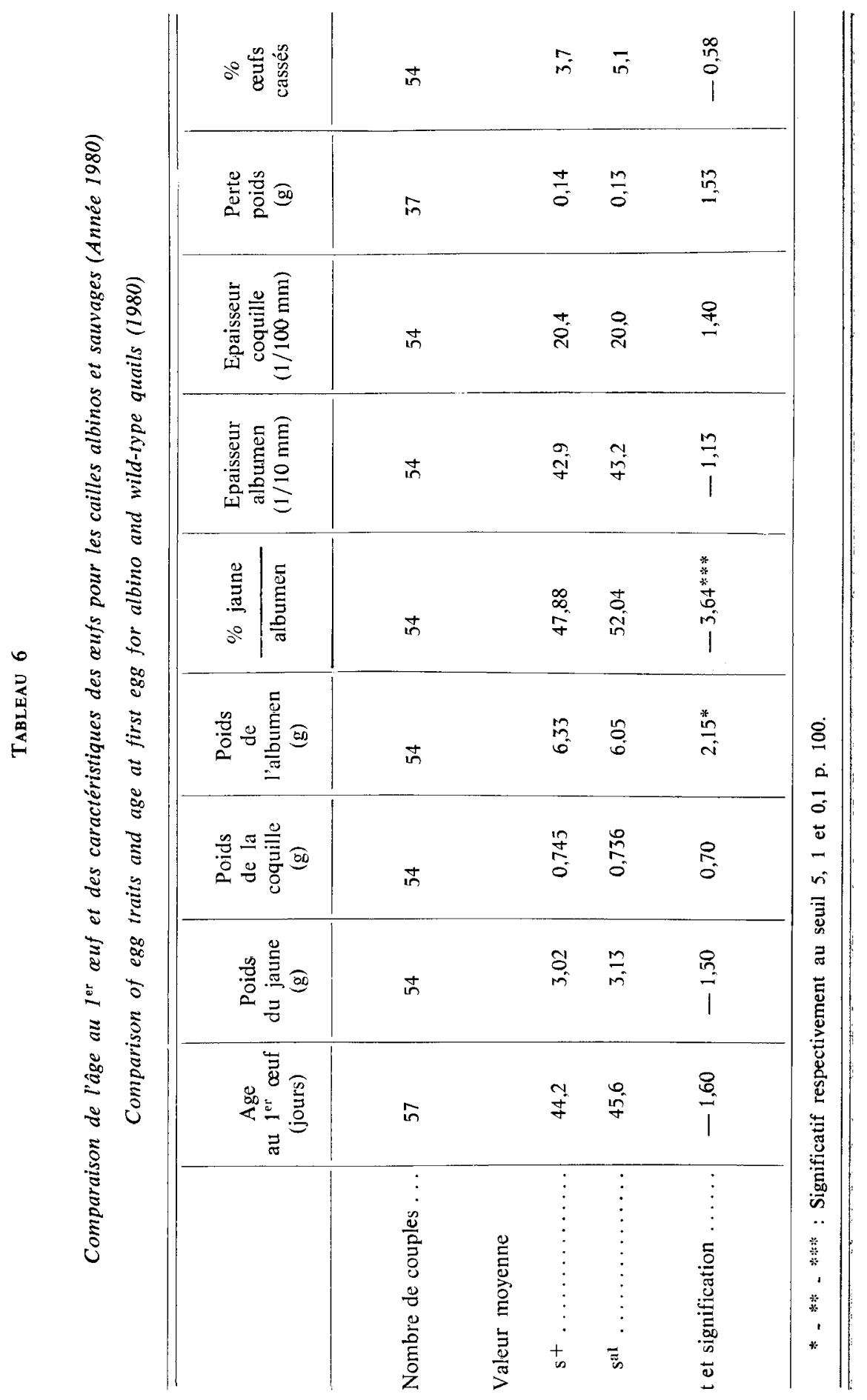




\section{TABLEAU 7}

Note d'état du plumage des cailles adultes de type sauvage ou albinos

Score of plumage condition for albino and wild-type adult quails

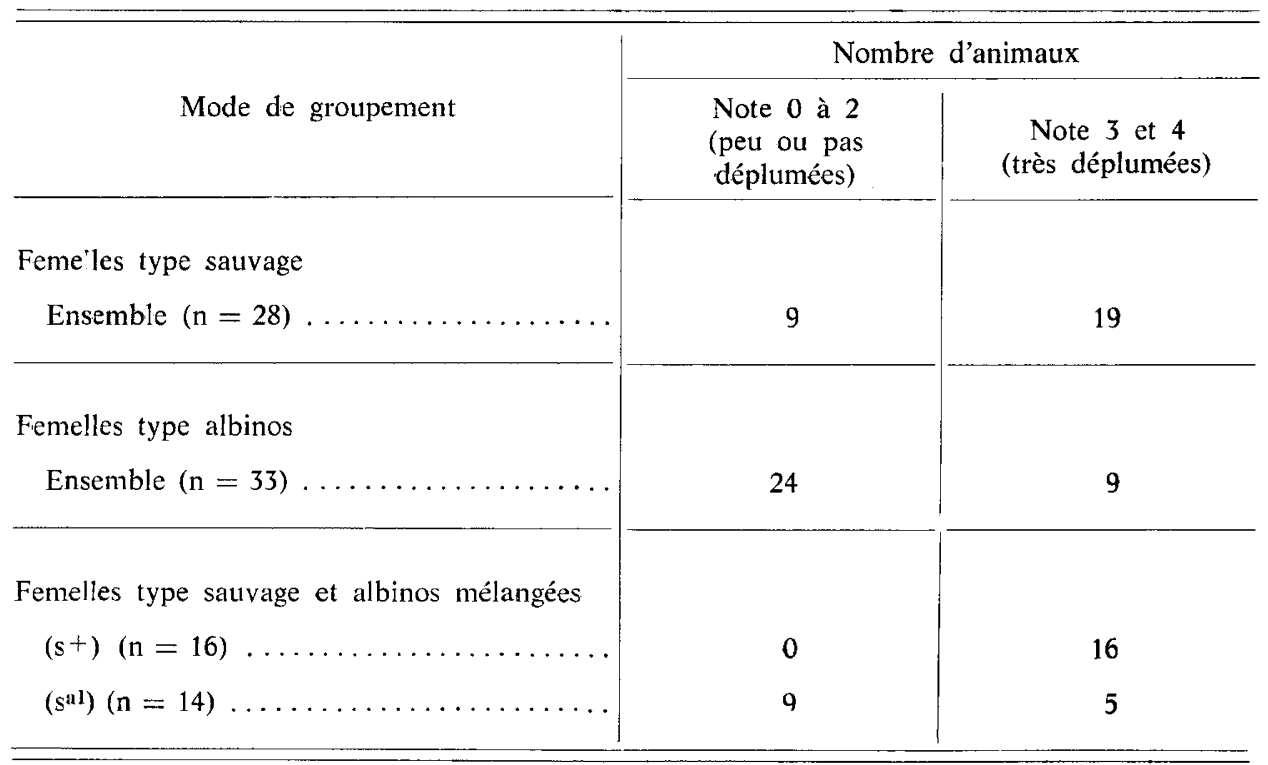

\section{Discussion et conclusions}

\section{Eclosion et mortalité juvénile}

Les données du tableau 2 relatives à la première éclosion de 1980 suggèrent un excès de phénotypes albinos parmi les embryons morts tardivement, par rapport à la proportion théorique de 1 albinos pour 3 zygotes de type sauvage en supposant un effectif égal des deux sexes. Cependant, le second lot ne manifeste pas cette tendance et le $\chi^{2}$ sur l'ensemble des deux n'atteint pas le seuil $5 \mathrm{p}$. cent de signification. Une vérification ultérieure sur des nombres plus importants serait donc nécessaire. D'autre part, rappelons qu'il ne s'agit que de la mortalité tardive (3 derniers jours d'incubation), stade auquel la détermination du phénotype des embryons est pratiquement réalisable.

La mortalité juvénile (avant 5 semaines) dont nous avons pu tenir compte, relative à la seconde éclosion de 1980, montre qu'à ce stade les albinos sont très nettement désavantagés par rapport aux individus sauvages. La différence, surtout accentuée dans les premiers jours, s'estompe graduellement ensuite. Après 5 semaines, la mortalité observée est partout faible. On ne peut donner pour l'instant d'explication sûre. La localisation exclusive des pertes au début de la vie suggère la possibilité d'une 
différence de comportement associée au gène albinos : retard à trouver la nourriture dans les premiers jours après l'éclosion, ou infériorité dans une compétition sociale ? A propos du gène similaire lié au sexe chez la Poule, un handicap des oiseaux albinos du point de vue de la vision, a été suggéré (Champion, 1958; WerretT et al., 1959), mais ce n'est pas l'opinion de Mueller \& HutT (1941) et de HutT \& COLE (1973).

\section{Croissance}

Le tableau 3 montre que les femelles albinos ont un poids corporel moyen significativement inférieur à celui des sauvages à tous les âges. D'autre part, la différence de poids moyen entre les deux génotypes diminue régulièrement avec le temps. Ceci est encore plus marqué si l'on considère le rapport de cette différence à la moyenne de poids des deux génotypes : sa valeur exprimée en p. 100 aux quatre âges successifs où une pesée a été faite est respectivement 13,$6 ; 9,0 ; 4,2$ et 2,7. Le retard de croissance des albinos est donc partiellement compensé avec l'âge. Comme pour la mortalité en début d'élevage, on peut se demander si ce retard initial des cailleteaux albinos ne correspond pas à une sous-consommation alimentaire par suite de facteurs de compétition sociale ou à cause d'une gêne de vision dans nos conditions expérimentales. On peut d'autre part noter, chez la Poule, l'opinion de WERRETT et al. (1959) selon laquelle les poussins albinos sont de plus petite taille à l'éclosion et semblent avoir une croissance retardée.

\section{Activité}

Les deux années (tableau 4), le temps de latence est significativement plus grand pour les cailles albinos comparées aux cailles de type sauvage. A l'inverse, le nombre de «tops » est plus faible pour le premier génotype, et la différence est hautement significative en 1980 et pour le total général. Les cailles albinos apparaissent donc moins «actives» que leurs sœurs pigmentées. D'après Faure (1975), le test en open-field reflète aussi des différences d'émotivité, les poussins moins actifs étant plus émotifs. Dans le cas présent, cependant, on peut penser encore à une moins bonne vue des albinos inhibant leurs déplacements dans un milieu inconnu. La vision pourrait alors être le facteur causal commun réduisant l'activité et la consommation alimentaire juvénile et contribuant à la mortalité précoce.

Il est intéressant de noter que des souris albinos, obtenues à partir d'une mutation, ont été trouvées moins actives que des souris normales dans un open-field (Fuller, 1967).

\section{Caractères de ponte et poids moyen des œufs}

L'âge au $1^{\text {er }}$ œuf n'est pas significativement différent pour les cailles albinos et celles de type sauvage (tableau 6), malgré le retard de croissance des premières. Il n'y a pas non plus de différence significative entre génotypes pour le nombre d'œufs (tableau 5). Il en est de même de la masse totale d'œufs pondus. Toutefois, la durée du contrôle ( 28 jours) est peut-être insuffisante pour tirer une conclusion défi- 
nitive quant à l'ensemble de la période de ponte. Par contre, les albinos pondent des œufs un peu moins lourds en moyenne que les femelles sauvages. Le résultat, de même sens les deux années, est significatif en 1980 et au total. Cette différence est attribuable selon toute vraisemblance au poids corporel resté moindre pour les albinos durant la période de contrôle de ponte (65 à 93 jours d'âge). Dans l'ensemble, nous trouvons entre le poids corporel moyen et le poids moyen des œufs dans cette période une corrélation de $+0,53$.

\section{Consommation et efficacité alimentaire des pondeuses}

Le tableau 5 montre que les cailles albinos ont une consommation alimentaire inférieure à celle des cailles sauvages. L'écart observé est dû en partie à la différence de poids corporel moyen durant le contrôle, mais non totalement. En effet, la variable $\mathrm{R}$, consommation alimentaire corrigée pour les différences de poids, gain de poids et production d'œufs, est plus faible pour les cailles albinos que pour celles à pigmentation normale, de façon très hautement significative au total. En pourcentage de la consommation moyenne de l'ensemble des deux génotypes, la différence de consommation observée (variable $\mathrm{O}$ ) représente $5,8 \mathrm{p} .100$ et celle portant sur la consommation résiduelle (variable R) représente 3,8 p. 100 sur le total des deux années. Indépendamment de leur taille, les cailles albinos ont donc une efficacité alimentaire meilleure que les normales.

Les tests d'activité (tableau 4) peuvent suggérer une dépense énergétique moindre des femelles albinos associée à une fréquence plus faible de leurs déplacements, dans la mesure où les différences d'activité persistent avec l'âge. Divers auteurs ont essayé d'évaluer le coût énergétique de l'activité et sa répercussion sur l'efficacité alimentaire, et concluent à un effet significatif de la première, notamment chez la Poule pondeuse (par exemple : Balnave, 1974 ; Filmer, 1974 ; van Kampen, 1976). Cependant, sur l'ensemble de nos données sur une base intra-année et génotype, la corrélation phénotypique entre la variable $R$ et, d'autre part, le temps de latence et le nombre de 《tops» est respectivement $-0,01$ et $+0,15$ (non significatif). Il est donc difficile de conclure pour l'instant; la corrélation au niveau phénotypique peut différer de celle existant au niveau génétique. Quant à cette dernière, les effectifs disponibles ne permettaient pas de l'évaluer de façon satisfaisante. Par ailleurs, d'autres hypothèses pourraient être émises : différence d'intensité du métabolisme ou de thermolyse, associées au gène $s^{\mathrm{al}}$. Notons en tout cas que pendant notre contrôle en cages individuelles, aucune détérioration appréciable du plumage, avec zones dénudées, n'est apparue. Ce phénomène n'a donc pu contribuer à la différence d'efficacité alimentaire observée.

\section{Caractéristiques des oufs}

Les différences entre génotypes dans les poids des composantes de l'œuf mesurés en 1980 (tableau 6) vont de pair avec la différence du poids moyen de l'œuf entier. Seule la différence dans le poids de l'albumen est significative. Plus intéressant est le fait que le rapport du poids du jaune à celui de l'albumen est très significativement plus élevé chez les femelles albinos que chez celles de type sauvage. Ceci n'apparaît pas comme le simple reflet de l'écart du poids moyen de l'œuf entre ces génotypes 
car la corrélation de ce dernier avec le rapport du poids du jaune à celui de l'albumen n'est pas significative dans notre échantillon.

Les autres caractéristiques (hauteur de l'albumen, épaisseur de coquille, perte de poids de l'œuf à $38{ }^{\circ} \mathrm{C}$, pourcentage d'œufs cassés) ne montrent pas d'effet significatif associé au génotype au locus albinos et n'appellent pas d'autres commentaires.

\section{Etat du plumage en cages de groupe}

Il est apparent, d'après le tableau 7, que l'état du plumage dans les cages collectives est meilleur, dans l'ensemble, pour les femelles albinos que pour leurs sæurs ou demi-sœurs à plumage pigmenté. Plus précisément, le $\chi^{2}$ de contingence corrigé entre les femelles albinos gardées seules dans une même cage et les femelles «sauvages 》 seules (note 0 à 2 contre note 3 ou 4) est égal à 7,66 (p<0,01). Pour les femelles des deux génotypes mélangées dans la même cage, le $\chi^{2}$ analogue est égal à $11,79(\mathrm{p}<0,001)$. Il semble y avoir, quel que soit le génotype mais surtout chez le type sauvage, un peu plus de cailles à plumage fortement détérioré dans la cage contenant les génotypes mélangés que dans l'ensemble des deux autres, mais le $\chi^{2}$ de contingence correspondant, égal à 3,79, n'atteint pas le seuil 5 p. 100 de signification. Ceci étant, il paraît légitime de comparer globalement (les trois cages étant réunies) les deux génotypes pour l'état de leur plumage. Le $\chi^{2}$ de contingence ainsi obtenu atteint la valeur élevée de 20,65 ( $\mathrm{p}<0,001$ ).

Si l'on considère les valeurs moyennes des notes d'emplumement, sans regroupement en deux classes, l'indication est la même. Dans les cages contenant un seul génotype la valeur moyenne des femelles de type sauvage est 2,79 contre 1,97 pour la moyenne des albinos. Dans la cage avec génotypes mélangés, les moyennes des femelles sauvages et albinos sont respectivement 3,50 et 1,64. Rappelons qu'en cages individuelles, jusqu'à l'âge de 93 jours, il n'y avait pas de détérioration importante du plumage. Celle-ci est apparue avec l'élevage en groupes, et a pris un développement considérable en l'espace de deux mois. Il resterait à vérifier dans un essai de plus longue durée, si une proportion appréciable des albinos maintient jusqu'en fin de ponte l'intégralité de son plumage, ou si elles atteignent le même état de détérioration que les sauvages avec seulement un décalage dans le temps. Par ailleurs, la présente expérience ne pouvait, quant à la cause de cette détérioration, tranche entre l'hypothèse de l'usure mécanique et celle d'un arrachement de plumes par les femelles voisines, car un facteur «âge» se superpose au passage de cages individuelles en cages collectives. Cependant, l'extension rapide des défectuosités du plumage à dater de l'élevage en groupes, suggère l'intervention d'un facteur «picage». Cette hypothèse a été présentée comme vraisemblable chez la Poule pondeuse, par exemple par Hughes \& Duncan (1972), Charles (1976).

Sur poules adultes en cages, nous avons montré (MERAT et al., 1979) qu'un meilleur état du plumage était associé à la présence de plusieurs gènes supprimant ou réduisant la pigmentation de celui-ci. Il serait intéressant d'étendre cette comparaison au gène albinos lié au sexe connu dans cet espèce. 


\section{Conclusion}

Le gène albinos lié au sexe apparaît, chez la Caille japonaise, en nette infériorité du point de vue de la sélection naturelle, étant donné l'accroissement de la mortalité des premiers jours, et peut-être de la mortalité en fin d'incubation, dont il s'accompagne. Du point de vue des performances zootechniques, il est défavorable à la croissance dans la phase juvénile. Le rapprochement causal suggéré pour ces effets, de même que pour la moindre activité des femelles albinos, avec un défaut possible de vision de ces dernières, fait penser qu'une modification de certains facteurs de l'élevage (nombre des mangeoires et abreuvoirs, éclairage...) pourrait être essayée pour atténuer ces inconvénients. Par contre, des effets favorables liés au gène $s^{\text {ull }}$ sont observés chez les femelles en ponte. L'efficacité alimentaire est améliorée, la consommation d'aliment étant réduite de 4 p. 100 à production d'œufs et poids corporel égaux. De plus, le poids corporel étant diminué, l'ingestion d'aliments, au total, est abaissée de près de 6 p. 100 au prix d'une diminution du poids moyen des œufs d'un peu moins de 3 p. 100, mais, semble-t-il, sans que leur nombre et leur masse totale soient abaissés. Au surplus, notre contrôle en cages individuelles conduit selon toute vraisemblance à sous-estimer l'effet favorable associé au gène $s^{\text {al }}$ sur l'efficacité alimentaire des femelles en ponte, car, dans ces conditions, les différences portant sur l'état du plumage, observées en cages collectives, ne se sont pratiquement pas manifestées. Or, selon les observations faites chez la Poule (par exemple : Charles, 1976 ; Emmans \& Charles, 1976 ; Wathes, 1976 ; Leeson \& MorriSON, 1978), la détérioration du plumage en cours de ponte est un facteur important de diminution de l'efficacité alimentaire, dû à une augmentation des déperditions caloriques par les zones déplumées.

Dans le seul contexte de la présente expérience, il est impossible a priori d'écarter l'hypothèse d'un linkage entre le gène $s^{\mathrm{al}}$ et un gène responsable des effets quantitatifs observés. Cependant, un rôle direct joué par le gène albinos lui-même nous paraît plus plausible, sous réserve de vérifications des hypothèses explicatives émises plus haut. Un autre argument en faveur du caractère pléiotropique des effets constatés pourrait être fourni par l'observation d'un parallélisme de ces derniers dans les deux espèces, Coturnix coturnix japonica et Gallus gallus.

A la suite de ces premiers résultats obtenus chez Coturnix, il sera intéressant de chercher si, chez la poule, des effets similaires sont associés au gène albinos lié au sexe qu'elle possède, spécialement quant à l'efficacité alimentaire pour la production d'œufs. Un indice supplémentaire encourageant peut être l'absence suggérée d'effets défavorables importants sur la survie des jeunes liés à ce type d'albinisme dans l'espèce Gallus gallus (Mueller \& Hutt, 1941 ; Hutt, 1973). De plus, selon HutT (1973), le gène $s^{\text {al }}$ de la poule est pratiquement utilisable pour le sexage à l'éclosion.

$$
\text { Reçu pour publication en mai } 1981 .
$$

\section{Remerciements}

Nous remercions Monsieur J.-J. LAuvergne (I.N.R.A., 78350 Jouy-en-Josas) et Monsieur B. LeclercQ (I.N.R.A., Station de Recherches avicoles, Centre de recherches de Tours, 37380 Nouzilly) de leurs utiles suggestions et remarques concernant ce manuscrit. 


\section{Summary \\ Quantitative effects associated with the sex-linked albino gene in the Japanese Quail}

In two successive years (1979 and 1980) female quails (Coturnix coturnix japonica) of two genotypes were compared : wildtype v. sex-linked albino ( $s^{\text {al }}$ gene). The latter were fullor half-sisters of the former. Traits compared included survival rate at the end of incubation and after hatching, activity in an open-field, and quantitative measurements concerning growth rate, egg production and egg traits, food efficiency in individual cages, and plumage condition after transfer to group cages.

Albinos compared to colored quails showed considerably higher mortality after hatching. This is limited to the first weeks of age and disappears after 5 weeks. Initial growth is retarded, but the difference in body weight (absolute or in p. 100 of the overall mean) of the two genotypes decreases till the adult age (93 days). 5- weeks old albino quails are highly significantly less active (latency time and number of tops). Age at first egg, egg number and egg mass laid during 28 days do not differ for the albino and wildtype genotypes. Conversely, food consumption of albino layers is about 6 p. 100 less than that of wild-type females. Expressed as deviation from a multiple regression on body weight, body weight variation and egg mass it remains about 4 p. 100 inferior for the albino type. Mean egg weight is slightly lower and percent yolk is superior for this genotype. On the other hand, after 2 months in group cages, albino quails on the average have better plumage condition than their pigmented full- or half-sisters, which is likely to cause an additional difference in food efficiency between the two genotypes as compared with result in individual cages.

\section{Références bibliographiques}

Balnave D., 1974. Biological factors affecting energy expenditure. In: Energy requirements of poultry, pp. 25-46. Ed. T.R. Morris, B.M. Freeman. Br. Poult. Sci. Ltd Edinburgh.

ByerLy T.C., 1941. Feeds and other costs of producing market eggs. Univ. Maryland. Agric. Exp. Sta. Bull., $\mathrm{n}^{\circ}$ A1.

Champion L.R., 1958. The inheritance of imperfect albinism in the Fowl. Quat. Bull. Michigan agric. Exp. Sta., 41, 237-245.

Charles D.R., 1976. The economic importance of feathering. Gleadthorpe Poultry Booklet, pp. 25-27. Mandfield, Notts, Gleadthorpe Experimental Husbandry Farm.

Emmans G.C., Charles D.R., 1976. Climatic environment and poultry feeding in practice. In: Nutrition and the climatic environment, pp. 31-49. Edit. Haresign W., Swan H., Lewis D., Butterworths, London.

Faure J.M., Folmer J.C., 1975. Etude génétique de l'activité précoce en open-field du jeune poussin. Ann. Génét. Sél. anim., 7, 123-132.

Filmer D.G., 1974. Factors influencing food intake in practice. The laying fowl. Univ. Nottingham. Proc. Nutr. Conf. for feed Manufacturers, 7, 141-182.

Fuller J.L., 1967. Effects of the albino gene upon behaviour of mice. Anim. Behav., 15, 467-470.

Homma K., Jinno M., Sato K., Ando A., 1968. Studies on perfect and imperfect albinism in the Japanese Quail. Jpn. J. Zootech. Sci., 39, 348-352.

Hughes B.O., Duncan J.J.H., 1972. The influence of strain and environmental factors upon feather pecking and cannibalism in Fowls. Br. Poult. Sci., 13, 525-547.

Hutt F.B., Cole R.K., 1973. Identification of sex in chicks by the way of the gene sal. Poult. Sci., 52, 2044 (Abstr.).

Lauber J.K., 1964. Sex-linked albinism in the Japanese quail. Science, 146, 948-950. 
LEESON S., Morrison W.D., 1978. Effect of feather cover on feed efficiency in laying birds. Poult. Sci., 57, 1094-1096.

Merat P., Bordas A., Coquerelle G., 1979. The relationship of several genes suppressing plumage color with body weight, food intake and feather loss of laying hens. Brit. Poult. Sci., 20, 587-594.

Mueller C.D., Hutt F.B., 1941. Genetics of the Fowl - 12 - Sex-linked imperfect albinism. J. Hered., 32, 71-80.

Snedecor G.W., Cochran W.G., 1967. Statistical methods. Iowa State University Press, Ames.

Van Kampen M., 1976. Activity and energy expenditure in laying hens. 2. The energy cost of exercise. J. agric. Sci., 87, 81-84.

WATHES C.M., 1976. The effect of feathering on heat loss of the laying fowl. Gleadthorpe Poultry Booklet, pp. 22-24, Mansfield. Notts., Greadthorpe Experimental Husbandry Farm.

Werret W.F., Candy A.J., King J.O.F., Sheppard P.M., 1959. Semi albino : a third sexlinked allelomorph of Silver and Gold in the Fowl. Nature, 184, 480-482. 